\title{
STOCHASTIC SIGNAL DESIGN ON THE DOWNLINK OF A MULTIUSER COMMUNICATIONS SYSTEM
}

\author{
Mehmet Emin Tutay, Sinan Gezici, and Orhan Arikan \\ Department of Electrical and Electronics Engineering, Bilkent University, Ankara, Turkey \\ \{tutay, gezici, oarikan\}@ee.bilkent.edu.tr
}

\begin{abstract}
Stochastic signal design is studied for the downlink of a multiuser communications system. First, a formulation is proposed for the joint design of optimal stochastic signals. Then, an approximate formulation, which can get arbitrarily close to the optimal solution, is obtained based on convex relaxation. In addition, when the receivers employ symmetric signaling and sign detectors, it is shown that the maximum asymptotical improvement ratio is equal to the number of users, and the conditions under which that maximum asymptotical improvement ratio is achieved are presented. Numerical examples are provided to explain the theoretical results.
\end{abstract}

Index Terms- Multiuser, downlink, probability of error, stochastic signaling, randomization, minimax.

\section{INTRODUCTION}

Recently, the effects of randomizing transmitted signals, additive "noise", and jammer power have been investigated in various studies such as [1]-[7]. In [1], the stochastic signaling approach is considered by modeling transmitted signals in a binary communications system as random variables instead of deterministic quantities for each information symbol. It is shown that the probability of error is minimized when each signal is represented by a randomization of at most three different signal levels under second and fourth moment constraints. The results are extended in [2] to cases in which stochastic signals and detectors are jointly designed. In addition, [8] investigates the problem of joint detector randomization and stochastic signaling for minimum probability of error receivers. The effects of randomization are observed also in improving performance of suboptimal detectors and estimators by injecting "noise" to their observations [3]-[5], [9]. For example, additive noise that is a randomization between two different signal levels can increase detection probabilities of some suboptimal detectors under false-alarm constraints [3], [4]. The studies in [6] and [7] investigate the convexity properties of the average probability of error in the presence of additive white Gaussian noise (AWGN) when maximum likelihood (ML) detectors are employed at the receivers. Based on the convexity results, the cases in which power randomization can or cannot be useful for improving error performance are specified. In addition, optimal jammer power randomization strategies are proposed.

This research was supported in part by the National Young Researchers Career Development Programme (project no. 110E245) of the Scientific and Technological Research Council of Turkey (TUBITAK).
Motivated by the recent results that illustrate the improvements obtained via randomization [1]-[7], the aim of this study is to formulate a generic signal design problem for the downlink of a multiuser communications system in which the signal for each symbol of a user is modeled as a random variable. In other words, by adopting the stochastic signaling approach in [1], the aim is to jointly design stochastic signals for all symbols of all users in the downlink of a direct-sequence spread-spectrum (DSSS) system in order to optimize error performance for given receiver structures. A1though the stochastic signal design is performed for a single user system in [1], the joint stochastic signal design for multiple users has not been considered before. The main challenge in the joint stochastic signal design is that the signal of each user affects not only its own error performance but also the error performance of all other users via interference.

In this study, the downlink of a DSSS system is considered, and the joint design of stochastic signals is performed for all symbols of all users. The main contributions can be summarized as follows: (i) Joint stochastic signal design is performed in a multiuser system for the first time. (ii) In addition to the generic problem formulation, which needs to be solved via global optimization algorithms due to its nonconvex nature, an approximate convex solution is obtained based on convex relaxation. (iii) Although the theoretical results are obtained for generic detector structures at the receivers, specific results are obtained for sign detectors. Namely, it is shown that, in the interference limited case, the ratio between the maximum error probabilities of the optimal deterministic and optimal stochastic signaling approaches can be as high as the number of users. Also, numerical examples are provided to illustrate the improvements via the proposed stochastic signaling approach over the deterministic approaches.

\section{SYSTEM MODEL}

Consider the downlink of a multiuser DSSS binary communications system, in which the baseband model for the transmitted signal is given by

$$
p(t)=\sum_{k=1}^{K} S_{k}^{\left(i_{k}\right)} c_{k}(t)
$$

where $K$ is the number of users, $S_{k}^{\left(i_{k}\right)}$ denotes the signal of user $k$ for $i_{k} \in\{0,1\}$, and $c_{k}(t)$ is the pseudo-noise signal for user $k$. The pseudo-noise signals spread the spectra of users' signals and provide multiple-access capability [10]. Information intended for user $k$ is carried by signal $S_{k}^{\left(i_{k}\right)}$, which 


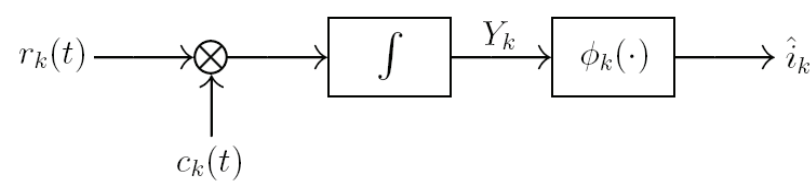

Fig. 1. Receiver structure for user $k$.

corresponds to bit 0 for $i_{k}=0$ and bit 1 for $i_{k}=1$. Signals are modeled to take real values, and they modulate the amplitudes of the real pseudo-noise signals. It is assumed that bit 0 and bit 1 are equally likely for all users, and the information bits for different users are independent.

The signal in (1) is transmitted to $K$ users, and the received signal at user $k$ is represented by

$$
r_{k}(t)=\sum_{l=1}^{K} S_{l}^{\left(i_{l}\right)} c_{l}(t)+n_{k}(t)
$$

for $k=1, \ldots, K$, where $n_{k}(t)$ denotes the noise at the receiver of user $k$, which is modeled as a zero-mean white Gaussian process with spectral density $\sigma_{k}^{2}$. It is assumed that the noise processes at different receivers are independent. Although a simple additive noise model is used in (2), multipath channels with frequency-flat fading can also be included in the considered model if perfect channel estimation is assumed at the receivers [1]. In that case, the average powers of the noise components in (2), equivalently, $\sigma_{k}^{2}$ terms, can be adjusted accordingly in order to take the channel conditions into account.

The receiver for user $k$ processes the signal in (2) as shown in Fig. 1. Specifically, the received signal $r_{k}(t)$ is correlated with the pseudo-noise signal for user $k, c_{k}(t)$, which effectively corresponds to a despreading operation, and then the correlator output is used by a generic detector in order to estimate the transmitted bit for user $k$. Based on (2), the correlator output for user $k$ can be expressed as

$$
Y_{k}=S_{k}^{\left(i_{k}\right)}+\sum_{\substack{l=1 \\ l \neq k}}^{K} \rho_{k, l} S_{l}^{\left(i_{l}\right)}+N_{k}
$$

for $k=1, \ldots, K$, where $\rho_{k, l} \triangleq \int c_{k}(t) c_{l}(t) d t$ denotes the crosscorrelation between the pseudo-noise signals for user $k$ and $l$ (it is assumed that $\rho_{k, k}=1$ for $k=1, \ldots, K$ ), and $N_{k} \triangleq \int n_{k}(t) c_{k}(t) d t$ is the noise component. It can be shown that $N_{1}, \ldots, N_{K}$ form a sequence of independent zero-mean Gaussian random variables with variances, $\sigma_{1}^{2}, \ldots, \sigma_{K}^{2}$, respectively. In (3), the first term corresponds to the desired signal component, the second term represents the multiple-access interference (MAI), and the last term is the noise component.

The correlator output in (3) is used by a generic detector (decision rule) $\phi_{k}$ to generate an estimate of the transmitted information bit, as shown in Fig. 1. Specifically, for a given correlator output $Y_{k}=y_{k}$, the bit estimate is denoted as

$$
\hat{i}_{k}=\phi_{k}\left(y_{k}\right)= \begin{cases}0, & y_{k} \in \Gamma_{k, 0} \\ 1, & y_{k} \in \Gamma_{k, 1}\end{cases}
$$

for $k=1, \ldots, K$, where $\Gamma_{k, 0}$ and $\Gamma_{k, 1}$ denote the decision regions for bit 0 and bit 1 , respectively, and they form a partition of the observation space [11]. In the next section, theoretical results are obtained for generic detectors at the receivers; that is, $\phi_{k}$ 's can be arbitrary decision rules.

\section{OPTIMAL STOCHASTIC SIGNAL DESIGN}

Conventionally, a deterministic signal value is transmitted for each bit of a given user; that is, $S_{k}^{\left(i_{k}\right)}$ in (1) are modeled as deterministic quantities. In this study, we adopt the stochastic signaling framework [1], and model signals $S_{k}^{\left(i_{k}\right)}$ in (1) as random variables. Let $S$ denote the vector of random variables corresponding to signals of all users; that is,

$$
\boldsymbol{S}=\left[S_{1}^{(0)} S_{1}^{(1)} S_{2}^{(0)} S_{2}^{(1)} \cdots S_{K}^{(0)} S_{K}^{(1)}\right]
$$

and let $p_{S}$ represent the probability density function (PDF) of $S$. Then, we formulate the stochastic signal design problem for multiuser downlink as follows:

$$
\begin{aligned}
& \min _{p_{S}} \max _{k \in\{1, \ldots, K\}} \mathrm{P}_{k} \\
& \text { subject to } \mathrm{E}\left\{\int|p(t)|^{2} d t\right\} \leq A
\end{aligned}
$$

where $\mathrm{P}_{k}$ denotes the average probability of error for user $k$, $p(t)$ is as in (1), and $A$ is a constraint on the average power of the transmitted signal. In other words, the aim is to find the optimal PDF of the signals that minimizes the maximum of the average probabilities of error under a constraint on the average transmitted power. The minimax approach is adopted for fairness [12]; that is, for preventing scenarios in which the average probabilities of error are very low for some users whereas they are (unacceptably) high for others.

To express the optimization problem in (6)-(7) more explicitly, we first manipulate the constraint in (7) based on (1).

$\mathrm{E}\left\{\int|p(t)|^{2} d t\right\}=\sum_{k=1}^{K} \sum_{l=1}^{K} \rho_{k, l} \mathrm{E}\left\{S_{k}^{\left(i_{k}\right)} S_{l}^{\left(i_{l}\right)}\right\}=\mathrm{E}\{H(\boldsymbol{S})\}$

where

$$
H(\boldsymbol{S}) \triangleq \sum_{k=1}^{K} \sum_{l=1}^{K} \rho_{k, l} S_{k}^{\left(i_{k}\right)} S_{l}^{\left(i_{l}\right)} .
$$

In some scenarios, symmetric signaling is used, i.e., signals are selected as $S_{k}^{(0)}=-S_{k}^{(1)}$ for $k=1, \ldots, K$. In that case, $\mathrm{E}\left\{S_{k}^{\left(i_{k}\right)} S_{l}^{\left(i_{l}\right)}\right\}=\mathrm{E}\left\{\left|S_{k}^{(1)}\right|^{2}\right\}$ if $k=l$ and $\mathrm{E}\left\{S_{k}^{\left(i_{k}\right)} S_{l}^{\left(i_{l}\right)}\right\}=$ 0 if $k \neq l$. Then, $H(\boldsymbol{S})$ becomes $H(\boldsymbol{S})=\sum_{k=1}^{K}\left|S_{k}^{(1)}\right|^{2}$.

Next, the average probability of error for user $k, \mathrm{P}_{k}$, is obtained, after some manipulation, as follows:

$$
\mathrm{P}_{k}=\mathrm{E}\left\{G_{k}(\boldsymbol{S})\right\}
$$

where the expectation is over the random vector $S$ in (5), and $G_{k}(\boldsymbol{S})$ is defined as

$$
\begin{gathered}
G_{k}(\boldsymbol{S}) \triangleq \frac{1}{2^{K}} \sum_{m \in\{0,1\}} \sum_{\mathbf{i}_{k} \in\{0,1\}^{K-1}} \mathrm{P}\left\{\left(N_{k}+S_{k}^{(m)}\right.\right. \\
\left.\left.+\sum_{\substack{l=1 \\
l \neq k}}^{K} \rho_{k, l} S_{l}^{\left(i_{l}\right)}\right) \in \Gamma_{k, 1-m}\right\} .
\end{gathered}
$$


The probabilities in (11) are calculated according to the PDF of $N_{k}$ for given values of $S_{k}^{\left(i_{k}\right)}$ 's, and $\mathbf{i}_{k}$ is defined as $\mathbf{i}_{k} \triangleq$ $\left[i_{1} \cdots i_{k-1} i_{k+1} \cdots i_{K}\right]$. Based on (8) and (10), the optimization problem in (6)-(7) can be stated as

$$
\begin{aligned}
\min _{p_{S}} & \max _{k \in\{1, \ldots, K\}} \mathrm{E}\left\{G_{k}(\boldsymbol{S})\right\} \\
& \text { subject to } \mathrm{E}\{H(\boldsymbol{S})\} \leq A .
\end{aligned}
$$

The optimization problem in (12)-(13) can be quite complex in its current form since it is nonconvex in general and requires optimization over all possible PDFs for a random vector of size $2 K$ (see (5)). ${ }^{1}$ Therefore, it is desirable to obtain a convex version of the problem, which is easier to solve and can get arbitrarily close to the optimal solution of (12)(13). In the following, such an approximate formulation of the problem is derived based on convex relaxation [13].

First, consider a set of possible signal values for $S$ in (5) and denote them as $\mathbf{s}_{1}, \ldots, \mathbf{s}_{N_{m}}$. Then, the signal PDF is approximately modeled as

$$
p_{\boldsymbol{S}}(\mathbf{x}) \approx \sum_{j=1}^{N_{m}} \lambda_{j} \delta\left(\mathbf{x}-\mathbf{s}_{j}\right)
$$

where $\sum_{j=1}^{N_{m}} \lambda_{j}=1, \lambda_{j} \geq 0$ for $j=1, \ldots, N_{m}$, and $\mathbf{s}_{1}, \ldots, \mathbf{s}_{N_{m}}$ are known signal values. Then, the approximate version of (12)-(13) can be formulated as follows:

$$
\begin{aligned}
\min _{\boldsymbol{\lambda}} & \max _{k \in\{1, \ldots, K\}} \boldsymbol{\lambda}^{T} \mathbf{g}_{k} \\
& \text { subject to } \quad \boldsymbol{\lambda}^{T} \mathbf{h} \leq A, \quad \boldsymbol{\lambda}^{T} \mathbf{1}=1, \quad \boldsymbol{\lambda} \geq \mathbf{0}
\end{aligned}
$$

where $\boldsymbol{\lambda} \triangleq\left[\lambda_{1} \cdots \lambda_{N_{m}}\right], \mathbf{g}_{k} \triangleq\left[G_{k}\left(\mathbf{s}_{1}\right) \cdots G_{k}\left(\mathbf{s}_{N_{m}}\right)\right], \mathbf{h} \triangleq$ $\left[H\left(\mathbf{s}_{1}\right) \cdots H\left(\mathbf{s}_{N_{m}}\right)\right]$, and $\mathbf{0}$ and $\mathbf{1}$ denote vectors of zeros and ones, respectively. In other words, instead of considering all possible PDFs as in (12)-(13), a number of known signal values are considered, and the optimal weights, $\lambda$, corresponding to those signal values are searched for. In general, the solution of (15)-(16) provides an approximation to the optimal solution that is obtained from (12)-(13), and the approximation accuracy can be improved as much as desired by increasing $N_{m}$. In fact, if $\mathbf{s}_{1}, \ldots, \mathbf{s}_{N_{m}}$ contain all the possible signal values (e.g., for a digital system), then the solution of (15)-(16) becomes exact.

By defining an auxiliary variable $t$, an equivalent form of (15)-(16) can be obtained as follows:

$$
\begin{aligned}
\min _{t, \boldsymbol{\lambda}} & t \\
\text { subject to } & \boldsymbol{\lambda}^{T} \mathbf{g}_{k} \leq t, k=1, \ldots, K \\
& \boldsymbol{\lambda}^{T} \mathbf{h} \leq A, \quad \boldsymbol{\lambda}^{T} \mathbf{1}=1, \quad \boldsymbol{\lambda} \geq \mathbf{0} .
\end{aligned}
$$

It is noted that (17)-(19) corresponds to linearly constrained linear programming (LCLP). Therefore, it can be solved efficiently in polynomial time [13].

Remark 1: It is noted that the stochastic signal model in (14) corresponds to a randomization among $N_{m}$ signal values. In practice, randomization of signal values can be performed, for example, via time sharing by sending each signal

\footnotetext{
${ }^{1}$ The dimension of $S$ reduces to $K$ if symmetric signaling is employed.
}

value for a certain number of information bits in proportion to the probability of that signal value. It is important to note that the receivers do not need to know this randomization structure since the signal randomization is optimized by the transmitter for given detectors at the receivers of different users (see (4)).

Remark 2: In order to realize the proposed stochastic signaling approach in practice, the transmitter needs to know the noise powers at the receivers (or, the signal-to-noise ratios (SNRs) at the receivers, considering a flat-fading scenario, as discussed after (2)), which can be sent via feedback to the transmitter. Such a feedback is commonly available in multiuser systems for power control purposes [14]. In addition, if the signal randomization is implemented via time sharing, the channel conditions should be constant for a number of bit durations; hence, slowly fading channels are well-suited for stochastic signaling.

\section{SPECIAL CASE: SIGN DETECTORS}

In this section, stochastic signaling is studied in detail for symmetric signaling when sign detectors are employed at the receivers. Although sign detectors may not be optimal in the presence of interference [15], they facilitate simple implementation as they have low complexity and do not need any prior information about the interference. The use of sign detectors is justified also by the zero mean nature of the noise and interference (see (3)). It should be noted that the interference has zero mean since symmetric signaling and equally likely information bits are assumed. For these reasons, sign detectors are employed in many binary communications systems, such as in various wireless sensor network applications due to their low complexity and practicality.

For the sign detectors, the decision rules at the receivers (see (4)) become

$$
\hat{i}_{k}=\phi_{k}\left(y_{k}\right)= \begin{cases}0, & y_{k}<0 \\ 1, & y_{k}>0\end{cases}
$$

for $k=1, \ldots, K$. In the case of $y_{k}=0$, the detector decides for bit 0 or bit 1 with equal probabilities. Then, for symmetric signaling (i.e., $S_{k}^{(1)}=-S_{k}^{(0)}$ for $\left.k=1, \ldots, K\right), G_{k}(\boldsymbol{S})$ in (11) can be expressed, after some manipulation, as

$G_{k}(\boldsymbol{S})=\frac{1}{2^{K-1}} \sum_{\mathbf{i}_{k} \in\{0,1\}^{K-1}} Q\left(\frac{S_{k}^{(1)}+\sum_{l=1, l \neq k}^{K} \rho_{k, l} S_{l}^{\left(i_{l}\right)}}{\sigma_{k}}\right)$

In order to provide intuitions about the performance of stochastic signaling in MAI limited scenarios, an asymptotical analysis is performed as $\sigma_{k} \rightarrow 0$ for $i=1, \ldots, K$. In this case, $G_{k}(\boldsymbol{S})$ in (21) is given by

$G_{k}(\boldsymbol{S})=\frac{1}{2^{K-1}} \sum_{\mathbf{i}_{k} \in\{0,1\}^{K-1}} u\left(-S_{k}^{(1)}-\sum_{l=1, l \neq k}^{K} \rho_{k, l} S_{l}^{\left(i_{l}\right)}\right)$

where $u(\cdot)$ represents the unit step function defined as $u(x)=$ 1 for $x>0, u(x)=0.5$ for $x=0$ and $u(x)=0$ for $x<0$.

Next, the aim is to compare the performance of the optimal stochastic and deterministic signaling approaches for 
sign detectors in the absence of noise. In the optimal deterministic signaling approach, the signals are modeled as deterministic values; that is, the PDF of $\boldsymbol{S}$ in (5), $p_{\boldsymbol{S}}$, is expressed as $p_{\boldsymbol{S}}(\mathbf{x})=\delta(\mathbf{x}-\mathbf{s})$. Then, the optimization problem in (12)(13) reduces to the optimal deterministic signaling problem:

$$
\min _{\mathbf{s}} \max _{k \in\{1, \ldots, K\}} G_{k}(\mathbf{s}) \text { subject to } H(\mathbf{s}) \leq A \text {. }
$$

Assume without loss of generality that signals $S_{k}^{(1)}$ are positive. Then, it is observed that both the optimal stochastic and deterministic signaling approaches can achieve zero probability of error if there exists a signal vector $\boldsymbol{S}$ such that ${ }^{2}$

$$
S_{k}^{(1)}>\sum_{l=1, l \neq k}^{K}\left|\rho_{k, l}\right| S_{l}^{(1)}, \forall k \in\{1, \ldots, K\} .
$$

This condition follows from (22) since it guarantees that the argument of the unit step function is negative for all signal combinations (recalling that $S_{l}^{(0)}=-S_{l}^{(1)}$ as symmetric signaling is considered).

The condition in (24) corresponds to scenarios in which MAI is not significant and no error floor occurs due to interference. However, this condition may not be satisfied in certain cases and the MAI can be significant. For those cases, it is important to quantify the maximum amount of improvement that can be achieved via stochastic signaling over deterministic signaling. Let $\mathrm{P}_{\text {stoc }}$ denote the minimum value of the maximum probability of error corresponding to the optimal stochastic signaling, which is obtained as the solution of (12)-(13). In addition, let $\mathrm{P}_{\text {det }}$ denote the minimum value of the maximum probability of error for optimal deterministic signaling, which is obtained from (23). Then, the following proposition specifies the maximum asymptotical improvement due to stochastic signaling.

Proposition 1: Suppose there exist no signal values that satisfy (24). Then, for sign detectors and symmetric signaling, the maximum asymptotical improvement ratio is equal to the number of users. That is,

$$
\lim _{\sigma_{1}, \ldots, \sigma_{K} \rightarrow 0} \frac{\mathrm{P}_{\text {det }}}{\mathrm{P}_{\text {stoc }}} \leq K .
$$

Also, the maximum asymptotical improvement ratio, $K$, is achieved if there exist signal values such that

$$
\begin{gathered}
S_{k}^{(1)}>\sum_{l=1, l \neq k}^{K}\left|\rho_{k, l}\right| S_{l}^{(1)}, \forall k \in\{1, \ldots, K\} \backslash\left\{k^{*}\right\} \text { and } \\
-2 \min _{l \in\{1, \ldots, K\} \backslash\left\{k^{*}\right\}}\left\{\left|\rho_{k^{*}, l}\right| S_{l}^{(1)}\right\}< \\
S_{k^{*}}^{(1)}-\sum_{l=1, l \neq k^{*}}^{K}\left|\rho_{k^{*}, l}\right| S_{l}^{(1)}<0
\end{gathered}
$$

for any $k^{*} \in\{1, \ldots, K\}$.

The proof is omitted due to the space limitation.

Proposition 1 states that in interference-limited scenarios, the maximum average probability of error can be reduced by

\footnotetext{
${ }^{2}$ It can be assumed without loss of generality that $\boldsymbol{S}$ satisfies the power constraint in (13) since scaling the signal vector $\boldsymbol{S}$ by any positive number does not affect the inequalities in (24).
}

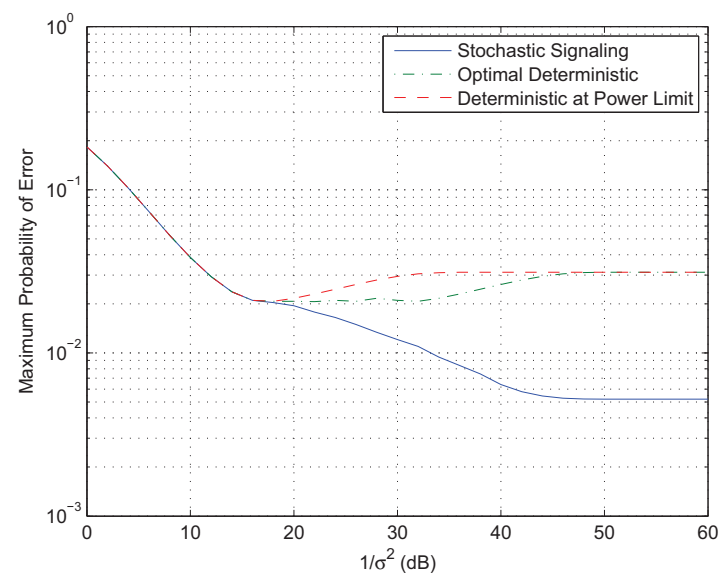

Fig. 2. Maximum probabilities of error versus $1 / \sigma^{2}$ for $K=$ $6, \rho_{k, l}=0.21$, for all $k \neq l$, and $A=6$.

a factor of up to $K$ via stochastic signaling. The main reason behind this improvement is the inherent randomization operation that is performed in the stochastic signaling approach. By employing randomization among multiple different signal vectors, the average probabilities of error for different users can be equalized to a certain extent, which can reduce the maximum value of the average probabilities of error.

\section{NUMERICAL RESULTS AND CONCLUSIONS}

In this section, simulations are performed in order to compare the performance of the stochastic signaling approach against various deterministic signaling approaches. Namely, the following techniques are investigated in the simulations:

Stochastic Signaling: Stochastic signals are designed based on the formulation in (17)-(19).

Optimal Deterministic Signaling: Deterministic signals are designed based on (23).

Deterministic Signaling at Power Limit: Instead of obtaining optimal deterministic signals from (23), one can also consider a deterministic signaling scheme which equalizes signal-to-interference-plus-noise ratios (SINRs) at different receivers, and utilizes all the available power at the transmitter [16]. For symmetric deterministic signaling, the SINR at the receiver of user $k$ can be expressed as $\operatorname{SINR}_{k}=$ $\left|S_{k}^{(1)}\right|^{2} /\left(\sum_{l \neq k} \rho_{k, l}^{2}\left|S_{l}^{(1)}\right|^{2}+\sigma_{k}^{2}\right)$. In the deterministic signaling at the power limit approach, $S_{1}^{(1)}, \ldots, S_{k}^{(K)}$ are chosen such that $\operatorname{SINR}_{1}=\cdots=\operatorname{SINR}_{K}$ and $\sum_{k=1}^{K}\left|S_{k}^{(1)}\right|^{2}=A$.

In the simulations, equally likely information bits are assumed, and symmetric signaling is considered. Also, the users employ sign detectors at the receivers, and the standard deviations of the noise at the receivers are taken to be equal, that is, $\sigma_{k}=\sigma$, for $k=1, \ldots, K$. In addition, without loss of generality, $\rho_{k, l}$ in (3) is set to one for $k=l$; that is, $\rho_{k, k}=1$ for $k=1, \ldots, K$.

First, a 6-user scenario is considered, that is, $K=6$, and the crosscorrelations between the pseudo-noise signals for different users are set to 0.21 ; i.e., $\rho_{k, l}=0.21$ for $k \neq l$. Also, the average power constraint $A$ in (7) is taken as 6 . In Fig. 2, the maximum probabilities of error are illustrated 


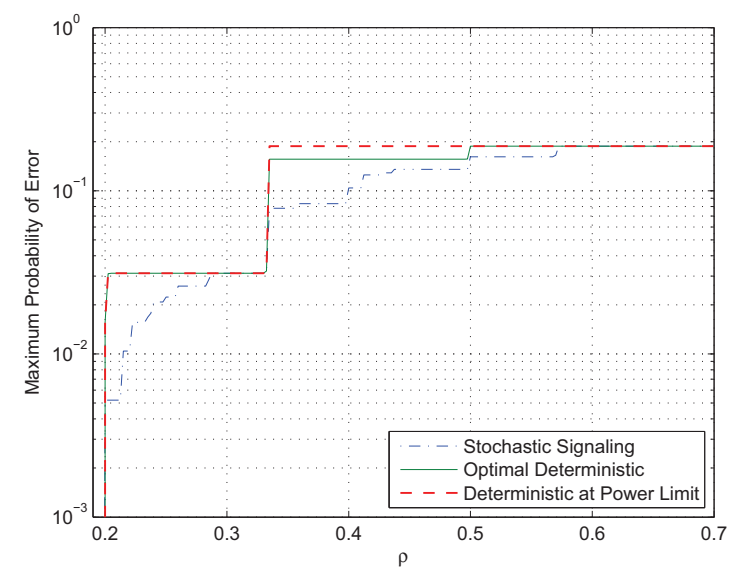

Fig. 3. Maximum probabilities of error versus $\rho$ for various signaling approaches, where $K=6, A=6$, and $\sigma=10^{-3}$.

for the stochastic signaling, optimal deterministic signaling, and deterministic signaling at the power limit approaches. In obtaining the stochastic signals via the convex relaxation approach, the signal for information bit 1 of each user is modeled to take values from 0 to 1.4 with an increment of $0.2 .^{3}$ Then, the optimal weights for these possible signal values are obtained from (17)-(19) via CVX: Matlab Software for Disciplined Convex Programming [17]. The use of a finite set of signal values can be justified by considering a digital system in which a number of bits are used to represent each signal value. In this scenario, a 4-bit representation can be considered as there are 8 possible signal values, $\{0,0.2,0.4,0.6,0.8,1,1.2,1.4\}$, for information bit 1 , and the negative of these values for information bit 0 . From Fig. 2, it is observed that the stochastic signaling approach outperforms the deterministic signaling approaches for small noise variances; that is, for MAI limited scenarios. Also, the optimal deterministic signaling approach achieves lower maximum probabilities of error than the optimal deterministic signaling at the power limit approach for medium range of $\sigma$ values. Another important observation from the figure is that, for small values of $\sigma$, the stochastic signaling approach achieves a 6 times improvement in the maximum probability of error compared to the optimal deterministic approach, as claimed in Proposition 1. In fact, it can be shown that the assumptions in the proposition are satisfied in this scenario.

In Fig. 3, the error probabilities of the different signaling approaches are plotted versus $\rho$, where $\rho_{k, l}=\rho$ for $k \neq l$. In addition, the other parameters are set to $A=6, K=6$, and $\sigma=10^{-3}$. It is observed that the stochastic signaling approach has lower error probabilities than the other approaches for $\rho \in[0.2,0.29]$ and $\rho \in[0.33,0.57]$. The improvement region and the amount of improvement depend on the relation among the system parameters. Also, the optimal deterministic signaling approach outperforms the deterministic signaling at the power limit approach for certain range of $\rho$. However, it does not provide significant improvements in general.

\footnotetext{
${ }^{3}$ Since symmetric signaling is considered, the possible signal values for bit 0 are from -1.4 to 0 with an increment of 0.2 .
}

\section{REFERENCES}

[1] C. Goken, S. Gezici, and O. Arikan, "Optimal stochastic signaling for power-constrained binary communications systems," IEEE Trans. Wireless Commun., vol. 9, no. 12, pp. 3650-3661, Dec. 2010.

[2] C. Goken, S. Gezici, and O. Arikan, "Optimal signaling and detector design for power-constrained binary communications systems over non-Gaussian channels," IEEE Commun. Letters, vol. 14, no. 2, pp. 100-102, Feb. 2010.

[3] H. Chen, P. K. Varshney, S. M. Kay, and J. H. Michels, "Theory of the stochastic resonance effect in signal detection: Part I-Fixed detectors," IEEE Trans. Sig. Processing, vol. 55, no. 7, pp. 3172-3184, July 2007.

[4] A. Patel and B. Kosko, "Optimal noise benefits in NeymanPearson and inequality-constrained signal detection," IEEE Trans. Sig. Processing, vol. 57, no. 5, pp. 1655-1669, 2009.

[5] H. Chen, P. K. Varshney, and J. H. Michels, "Noise enhanced parameter estimation," IEEE Trans. Sig. Processing, vol. 56, no. 10, pp. 5074-5081, Oct. 2008.

[6] M. Azizoglu, "Convexity properties in binary detection problems," IEEE Trans. Inform. Theory, vol. 42, no. 4, pp. 13161321, July 1996.

[7] S. Loyka, V. Kostina, and F. Gagnon, "Error rates of the maximum-likelihood detector for arbitrary constellations: Convex/concave behavior and applications," IEEE Trans. Information Theory, vol. 56, no. 4, pp. 1948-1960, April 2010.

[8] B. Dulek and S. Gezici, "Detector randomization and stochastic signaling for minimum probability of error receivers," IEEE Trans. Commun., vol. 60, no. 4, pp. 923-928, April 2012.

[9] M. D. McDonnell, "Is electrical noise useful?," Proceedings of the IEEE, vol. 99, no. 2, pp. 242-246, Feb. 2011.

[10] S. Verdu, Multiuser Detection, 1st ed. Cambridge, UK: Cambridge University Press, 1998.

[11] H. V. Poor, An Introduction to Signal Detection and Estimation, Springer-Verlag, New York, 1994.

[12] S. A. Grandhi, J. Zander, and R. Yates, "Constrained power control," Wireless Personal Communications, vol. 1, no. 4, pp. 257-270, 1995.

[13] S. Boyd and L. Vandenberghe, Convex Optimization, Cambridge University Press, Cambridge, UK, 2004.

[14] D. M. Novakovic and M. L. Dukic, "Evolution of the power control techniques for DS-CDMA toward $3 \mathrm{G}$ wireless communication systems," IEEE Communications Surveys, vol. 3, no. 4, pp. 2-15, 2000.

[15] S. Bayram and S. Gezici, "On the performance of singlethreshold detectors for binary communications in the presence of Gaussian mixture noise," IEEE Trans. Commun., vol. 58, no. 11, pp. 3047-3053, Nov. 2010.

[16] S. Koskie and Z. Gajic, "SIR-based power control algorithms for wireless CDMA networks: An overview," Dynamics of Continuous, Discrete and Impulsive Systems B: Applications and Algorithms, vol. 10-S, pp. 286-293, 2003.

[17] “CVX: Matlab software for disciplined convex programming, Version 1.21 (April 2011, Build 808),". 